\title{
Relation of Systemic-to-Pulmonary Artery Collateral Flow in Single Ventricle Physiology to Palliative Stage and Clinical Status
}

\author{
Ashwin Prakash, MD ${ }^{a, b,{ }^{*}}$, Rahul H. Rathod, MD ${ }^{a, b}$, Andrew J. Powell, MD ${ }^{a, b}$, Doff B. \\ McElhinney, MD ${ }^{a, b}$, Puja Banka, MD ${ }^{a, b}$, Tal Geva, MD ${ }^{a, b}$ \\ aDepartment of Cardiology, Children's Hospital Boston, Boston, Massachusetts. \\ bDepartment of Pediatrics, Harvard Medical School, Boston, Massachusetts.
}

\section{Abstract}

Systemic-to-pulmonary collateral arteries (SPCs) are common in patients with single- ventricle physiology, but their impact on clinical outcomes is unclear. The aim of this study was to use retrospective cardiac magnetic resonance data to determine the relation between SPC flow and palliative stage and clinical status in single-ventricle physiology. Of 116 patients, 78 were after Fontan operation (median age 19 years) and 38 were at an earlier palliative stage (median age 2 years). SPC flow was quantified as aortic flow minus total caval flow or total pulmonary vein flow minus total branch pulmonary artery flow. Median SPC flow/body surface area (BSA) was higher in the pre-Fontan group ( $1.06 \mathrm{vs} 0.43 \mathrm{~L} / \mathrm{min} / \mathrm{m} 2, \mathrm{p}<0.0001)$ and decreased nonlinearly with increasing age after the Fontan operation $(\mathrm{r} 2=0.17, \mathrm{p}<0.0001)$. In the Fontan group, patients in the highest quartile of SPC flow had larger ventricular end-diastolic volume/BSA ( $p<0.0001)$ and were older at the time of Fontan surgery $(\mathrm{p}=0.04)$, but SPC flow/BSA was not associated with heart failure symptoms, atrial or ventricular arrhythmias, atrioventricular valve regurgitation, the ventricular ejection fraction, or peak oxygen consumption. In multivariate analysis of all patients $(\mathrm{n}=116)$, higher SPC flow was independently associated with pre-Fontan status, unilateral branch pulmonary artery stenosis, a diagnosis of hypoplastic left-heart syndrome, and previous catheter occlusion of SPCs (model $\mathrm{r} 2=0.37, \mathrm{p}<0.0001$ ). In conclusion, in this cross-sectional study of single-ventricle patients, BSA-adjusted SPC flow was highest in pre-Fontan patients and decreased after the Fontan operation with minimal clinical correlates aside from ventricular dilation.

\begin{abstract}
Systemic-to-pulmonary collateral arteries (SPCs) are common in patients with singleventricle (SV) physiology, but their impact on clinical outcomes is unclear. ${ }^{1}$ Although some studies have postulated that SPC flow contributes to adverse outcomes such as abnormal ventricular mechanics, ${ }^{2}$ other reports have failed to show such associations. ${ }^{3}$ The lack of an accurate and readily applied technique to measure SPC flow in vivo has hindered research on this controversial topic. As a result, the amount of SPC flow and its clinical significance, time course, and optimal management in children with functional SVs remain unclear, with wide variation in management among centers. ${ }^{4}$ Recently, a cardiac magnetic resonance (CMR) technique for estimating SPC flow has been reported in small cohorts of young
\end{abstract}

\footnotetext{
*Corresponding author: Tel: 617-355-8366; fax: 617-734-9930. ashwin.prakash@ cardio.chboston.org (A. Prakash).
} 
patients with functional SV ${ }^{5-7}$ In the present study, we used CMR to quantify SPC flow in a cohort of functional SV patients and evaluated its relations with age, palliative stage, and clinical parameters.

\section{Methods}

A search of the CMR database at Children's Hospital Boston identified all patients with functional SV physiology who underwent CMR studies from January 2003 to June 2011. Patients with a complete set of flow measurements allowing calculation of SPC flow were included. Patients were divided into 2 groups: (1) those who had undergone Fontan operations (the Fontan group) and (2) those who were at any palliative stage before the Fontan operation (the pre-Fontan group). Because the required flow measurements were not performed routinely in the pre-Fontan group before 2009, inclusion was restricted to the period from January 2009 through June 2011. For patients who under- went >1 CMR examination during the study period, the examination with the most complete data set and/or closest in time to exercise stress testing (EST) was used for primary analyses. Those with significant ferromagnetic image artifacts, Fontan baffle leaks or fenestration visible on CMR or echocardiography, or significant ( $>3 \mathrm{~mm}$ in diameter) systemic-to-pulmonary venous collateral vessels visible on CMR or x-ray angiography were excluded. The study was approved by the Scientific Review Committee of the Department of Cardiology and by the Children's Hospital Boston Committee on Clinical Investigation.

Demographic and clinical data were abstracted from the medical records. Ventricular morphology was classified as left ventricular, right ventricular, or mixed (e.g., unbalanced atrioventricular canal). Ventricularv type was classified as mixed if the 2 ventricles had an end-diastolic volume (EDV) larger than a $\mathrm{z}$ score of $-4 .{ }^{8}$ Published normative data were used for calculation of $\mathrm{z}$ scores. ${ }^{9}$ Echocardiographic reports were reviewed, and the degree of atrioventricular valve regurgitation at the closest time to CMR was recorded.

CMR imaging was performed using 1.5-T whole-body scanners (Signa TwinSpeed, GE Healthcare, Milwaukee, Wisconsin; or Achieva, Philips Medical Systems, Best, The Netherlands). Electrocardiographically gated steady- state free precession cine magnetic resonance imaging in the vertical and horizontal ventricular long-axis planes and contiguous ventricular short-axis cine planes from the atrioventricular junction through the cardiac apex was performed. Ventricular volumes (indexed to body surface area [BSA]) and the ejection fraction were calculated using commercially available software (QMass MR; Medis, Leiden, The Netherlands) as previously described. ${ }^{10}$ In patients whose ventricular type was categorized as mixed, ventricular volumes were summed to calculate total EDV and endsystolic volume as previously described. ${ }^{10}$ Flow measurements were performed using an electrocardiographically gated through- plane cine phase-contrast magnetic resonance sequence. Target vessels included the ascending aorta, superior and inferior venae cavae, right and left pulmonary artery [PA] branches, and individual right and left pulmonary veins. Blood flow rates were calculated using commercially avail- able software (QFlow; Medis). SPC flow was quantified using 1 or both of the following methods as previously described and indexed to BSA ${ }^{5,6}$ : method 1: SPC flow = ascending aortic flow - (superior vena cava 
flow + inferior vena cava flow); method 2: SPC flow = total pulmonary vein flow - (right PA flow + left PA flow).

Contrast-enhanced 3-dimensional magnetic resonance angiography was performed after intravenous injection of $0.2 \mathrm{mmol} / \mathrm{kg}$ of gadopentate dimeglumine (Magnevist; Ber- lex, Seattle, Washington). Multiplanar reformatting was used to measure the diameters of the PA branches in orthogonal planes. Branch PA stenosis was defined as fulfill- ing the following 2 criteria: (1) $>50 \%$ narrowing relative to either a normal ipsilateral vessel segment or to the normal contralateral artery in cases in which there was diffuse hypoplasia and (2) $<30 \%$ flow through the involved PA.

EST data were available only in the Fontan group, be- cause most patients in the pre-Fontan group were too young to perform the test. Ramp cycle ergometry with assessment of gas exchange was performed to quantify percentage of predicted peak oxygen consumption for age and gender as previously described. ${ }^{11}$ Patients were referred for EST when clinically indicated, as determined by their primary cardiologists. Metabolic EST data were included if testing occurred within 1 year of the CMR study without intervening surgical or catheter intervention or change in clinical status and if the patient achieved maximal effort. A patient was considered to have reached maximal effort if he or she attained 1 or both of the following: respiratory exchange ratio $>1.09$ or $95 \%$ of predicted maximal heart rate. Patients with submaximal effort were excluded to avoid bias due to noncardiac factors. ${ }^{11}$

When available, conventional x-ray angiographic images were analyzed to assign SPC grade if all the following conditions were met: (1) angiography within 3 months of CMR, (2) no transcatheter occlusion of SPCs or cardiac surgical procedure between catheterization and CMR, and (3) adequate-quality angiography to assess overall SPC burden. Qualitative angiographic SPC grade ( 1 = none or mild, $2=$ moderate, $3=$ severe $)$ was assigned to each patient by a single observer (D.B.M.) blinded to CMR results using predefined criteria (Table 1).

Statistical analyses were performed using commercially available software (Stata version 10.0; StataCorp LP, College Station, Texas). To explore univariate associations of SPC flow/ BSA, the pre-Fontan and Fontan groups were each divided into "low-SPC flow" and "highSPC flow" categories using the group's median SPC flow as the cutoff. In the Fontan group, comparisons were also made between patients in the lowest and highest quartiles for SPC flow. Unadjusted associations were assessed using Student's $t$ test, Wilcoxon's rank-sum test, or Fisher's exact test, as appropriate. Multivariate linear regression analysis with forward selection was used to assess the statistical significance of associations between SPC flow estimated by CMR and clinical variables. Variables demonstrating univariate associations with $\mathrm{p}$ values $<0.10$ qualified for inclusion in the multivariate model. In a subset of the pre-Fontan group, agreement between methods 1 and 2 for quantifying SPC flow was assessed using Bland-Altman analysis. ${ }^{12}$ For all analyses, statistical significance was evaluated with respect to a type 1 error probability threshold of 0.05 . 


\section{Results}

Of the 307 patients with SV physiology (264 Fontan and 43 pre-Fontan) who underwent CMR during the study period, 116 (78 Fontan and 38 pre-Fontan) had data sufficient to estimate SPC flow by CMR and were included in the study. All CMR studies were requested by the patients' primary cardiologists for a variety of clinical indications, including but not limited to comprehensive evaluation be- fore a bidirectional Glenn shunt, evaluation of the pulmonary vasculature, and quantification of ventricular size and function. Demographic and clinical characteristics of the study patients are listed in Tables 2 and 3. In the Fontan group, patients included in the study were not different from those excluded with respect to their primary cardiac diag- noses, types of Fontan operations, and histories of superior cavopulmonary anastomosis before Fontan operation. How- ever, the included subjects were slightly older (age $21.8 \pm 10$ vs $18.8 \pm 10$ years, $p=0.02$ ), were more likely to have histories of atrial or ventricular arrhythmia ( $69 \%$ vs $48 \%, \mathrm{p}=0.001$ ), and heart failure symptoms ( $27 \%$ vs $13 \%, p=0.008$ ). In the pre-Fontan group, the number of excluded subjects was too small to compare with included subjects. The Fontan group was composed of predominantly young adults late after Fontan operation. Tricuspid atresia was the most common anatomic diagnosis. Most patients underwent initial palliation with systemic-to-PA shunts, but only a minority underwent intermediate volume-unloading procedures (usually a bidirectional Glenn shunt), reflecting clinical practice during the era of their surgical palliation. The most common type of Fontan procedure was a lateral tunnel. Only a small proportion (13\%) of subjects had un- dergone catheter-based embolization of SPCs.

The pre-Fontan group was composed of infants and young children, most (53\%) with hypoplastic left-heart syndrome (HLHS). Most patients in this group (74\%) had undergone superior cavopulmonary anastomosis, while the rest were at earlier palliative stages (26\%). The atrial septal defects were restricted in $16 \%$ of patients in preparation for possible biventricular repair. Approximately 1/3 (39\%) of the group had undergone previous catheterbased embolization of SPCs.

In the Fontan group, median SPC flow by method 1 was $0.43 \mathrm{~L} / \mathrm{min} / \mathrm{m} 2$ (interquartile range 0.13 to 0.79 ), accounting for a median of $15 \%$ (interquartile range $4 \%$ to $28 \%$ ) of ascending aortic flow. Given that data for calculating SPC flow by method 2 was available in only 2 patients, further analyses were performed using only method 1 .

In the pre-Fontan group using method $1(\mathrm{n}=35)$, median $\mathrm{SPC}$ flow was $1.06 \mathrm{~L} / \mathrm{min} / \mathrm{m} 2$ (interquartile range 0.65 to 1.77), accounting for a median of $24 \%$ (interquartile range $14 \%$ to $32 \%)$ of ascending aortic flow. Using method $2(n=24)$, median SPC flow was 1.26 $\mathrm{L} / \mathrm{min} / \mathrm{m} 2$ (inter- quartile range 1.0 to 1.82 ), accounting for a median of $29 \%$ (interquartile range $20 \%$ to $35 \%)$ of ascending aortic flow. There was a good correlation $(r=0.90, p$ $<0.0001$ ) and agreement (mean difference $0.17 \mathrm{~L} / \mathrm{min} / \mathrm{m} 2$, limits of agreement -1.1 to 0.77 ) between methods 1 and 2 in quantifying SPC flow/BSA $(n=22)$. Accordingly, for further analyses, data from method 2 were used as substitute when data from method 1 were missing. Median SPC flow in the pre-Fontan group using either method $(\mathrm{n}=38)$ was 1.06 $\mathrm{L} / \mathrm{min} / \mathrm{m} 2$ (interquartile range 0.65 to 1.77 ), account ing for a median of $26 \%$ (interquartile range $16 \%$ to $33 \%$ ) of ascending aortic flow. 
As seen in Figure 1, pre-Fontan patients had significantly higher SPC flow/BSA and SPC flow/ascending aortic flow compared to Fontan patients. Flow data for each group are listed in Table 4. Pre-Fontan patients demonstrated higher indexed systemic blood flow (superior vena cava flow + inferior vena cave flow/BSA) with higher BSA-adjusted flow in the superior vena cava but similar flow in the inferior vena cava, resulting in a higher proportion of caval return being composed of superior vena cava flow.

Because a higher proportion of the pre-Fontan group had HLHS compared to the Fontan group, we evaluated if this may have influenced the difference in SPC flow noted between the groups. Within each group, patients with HLHS had higher BSA-adjusted SPC flow compared to patients with other diagnoses, but these differences were not statistically significant ( $\mathrm{p}=0.10$ for the 2 groups). However, when BSA-adjusted SPC data for the 2 groups were combined for multivariate linear regression analysis, higher BSA-adjusted SPC flow was associated with a diagnosis of HLHS, even after controlling for Fontan status ( $\mathrm{p}=$ 0.02). When the BSA-adjusted SPC flow data for the 2 groups were combined and plotted against age (Figure 2), it was highest in the first several years of life, with a subsequent decrease until early adulthood, after which it remained relatively stable. The relation between SPC flow/BSA and age (analyzed as a continuous variable using linear regression and including quadratic terms for age) was nonlinear $\left(r^{2}=0.17, p<0.0001\right)$. In contrast, although absolute SPC flow did not vary significantly $\left(\mathrm{r}^{2}=0.03, \mathrm{p}=0.09\right)$ with increasing age, the trend suggested an increase during the first 3 years, after which absolute SPC flow remained stable (Figure 2).

Clinical outcomes for the Fontan group are listed in Table 2. The average percentage predicted peak oxygen consumption was subnormal and decreased with increasing age $\left(\mathrm{r}^{2}=\right.$ $0.11, p=0.03$ ), but heart failure symptoms were uncommon. Most patients had histories of atrial and/or ventricular arrhythmia, including nonsustained and sustained ectopy and tachyarrhythmias. Clinical outcomes could not be analyzed meaningfully in the pre-Fontan group due to the small and heterogenous sample.

In the Fontan group, patients with high (higher than the median) SPC flow had larger BSAadjusted ventricular EDVs than those with low (lower than the median) SPC flow (excluding patients with more than mild atrioventricular or semilunar valve regurgitation) (Table 2). The 2 groups did not differ significantly with respect to percentage predicted peak oxygen consumption, heart failure symptoms, the ventricular ejection fraction, and presence of more than mild atrioventricular valve regurgitation. Further univariate comparisons between patients in the lowest and highest quartiles of SPC flow/BSA showed that patients in the highest quartile were more likely to have larger ventricular EDV/BSA (Figure 3). Similar results were obtained when SPC flow was analyzed as a continuous variable using multivariate linear regression modeling. In these models, higher SPC flow/BSA was associated with larger ventricular EDV/BSA $\left(\mathrm{r}^{2}=0.19, \mathrm{p}=0.001\right)$ but not with percentage predicted peak oxygen consumption, heart failure symptoms, the ventricular ejection fraction, and presence of more than mild atrioventricular valve regurgitation. Of note, the magnitude of ventricular dilation was mild compared to normative values (mean EDV 115.2 $\pm 40 \mathrm{ml} / \mathrm{m}^{2}$ in the high-SPC flow subgroup vs $82.3 \pm 14.7 \mathrm{ml} / \mathrm{m}^{2}$ in healthy male volunteers). ${ }^{9}$ In the pre-Fontan group, analyses of associations with clinical factors were 
limited by the small sample size, but there was a trend toward higher ventricular EDV/BSA $(\mathrm{p}=0.09)$ in patients with higher than median SPC flow.

In the Fontan group, univariate comparisons between patients in the lowest and highest quartiles of SPC flow/ BSA showed that patients in the highest quartile were more likely to be older at the time of the Fontan operation (Figure 3), but this association was not significant by linear regression analysis. Analyses in the pre-Fontan group were limited by the small size of this group.

We then combined the Fontan and pre-Fontan groups $(\mathrm{n}=116)$ and used multivariate linear regression modeling to identify anatomic and surgical factors associated with BSA-adjusted SPC flow. In this analysis, factors independently associated with higher BSA-adjusted SPC flow included pre-Fontan status ( $\mathrm{p}=0.003$ ), unilateral branch PA stenosis on CMR as previously defined $(\mathrm{p}=0.001)$, a diagnosis of HLHS $(\mathrm{p}=0.036)$, and previous catheter occlusion of SPCs $(\mathrm{p}=0.001)$. This model explained $37 \%$ of the variability in BSA-adjusted SPC flow (model $\mathrm{r}^{2}=0.37, \mathrm{p}<0.0001$ ).

Angiographic SPC grade could be assessed in 26 of 38 pre-Fontan patients using the predefined criteria listed in Table 1. As seen in Figure 4, higher SPC flow/BSA was associated with higher angiographic SPC grade, but there was substantial overlap between grades. Angiographic SPC grade could not be assessed in the Fontan group, because adequate angiographic images were not available within 1 year of CMR in most patients.

\section{Discussion}

The hemodynamic impact and clinical ramifications of SPCs in patients with functional SVs have long been a topic of discussion and controversy. ${ }^{1-4}$ The lack of a reliable in vivo method for measurement of SPC flow has further hindered investigation into the subject. More recently, how- ever, 2 studies by Grosse-Wortmann et $\mathrm{al}^{5}$ and Whitehead et $\mathrm{al}^{6}$ described a CMR-based method to quantify SPC flow in patients who underwent staged palliation of functional SV. Although the focus of these reports was on outlining the method of SPC flow quantification, the clinical and long- term hemodynamic correlates of collateral flow were not elucidated in detail. The present study used the aforementioned CMR technique to measure SPC flow in a relatively large cohort of patients with functional SVs at different stages of their surgical palliation and explored the relations between the amount of collateral flow and clinical and hemodynamic parameters.

Our study found that SPC flow was highest in the pre- Fontan patients, especially during the first few years of life, and that collateral flow was lower with increasing patient age, mostly after Fontan completion. Moreover, beginning in the second decade of life, SPC flow tended to be rela- tively low and did not vary significantly with increasing age. The decrease in BSA-adjusted SPC flow with increasing age was due predominantly to a stable amount of collateral flow relative to increasing BSA with age. Importantly, we found in this cohort that aside from mild ventricular dilation, higher SPC flow was not associated with adverse clinical status after Fontan surgery. 
The reasons for the progressively lower SPC flow/BSA with increasing age are unclear. It is possible that an in- crease in systemic oxygen saturation after the Fontan oper- ation removes the stimulus for further growth of SPCs. Our finding that Fontan patients in the highest quartile of SPC flow/BSA underwent the Fontan operation at an older age supports this hypothesis. Relief of unilateral branch PA stenosis at the time of the Fontan operation may also con- tribute to the decrease in SPC flow/BSA. This is supported by a lower prevalence of unilateral branch PA stenosis (5\% vs 13\%) in the Fontan group.

Other factors that may contribute to the development of high SPC flow in early childhood remain incompletely de- fined. ${ }^{1,13,14}$ It is worth noting that none of these previous studies used a reliable method to quantify SPC flow. In our combined data set, including patients at all stages of palliation, we found associations between higher SPC flow and pre-Fontan status, the presence of unilateral branch PA stenosis, and a diagnosis of HLHS. The associations with pre-Fontan status and unilateral branch PA stenosis are intuitive and consistent with previous studies. However, the association with HLHS has not been previously described and deserves further study. Now that in vivo measurement of SPC flow is feasible, further investigation of factors that promote the formation and growth of SPCs in SV physiology is warranted.

Although SPC flow has been speculated to adversely affect long-term clinical outcomes, no data exist to support this hypothesis. In theory, SPC flow causes volume over- load of the systemic ventricle, which could contribute to adverse outcomes. In the present study, we evaluated various clinical and laboratory parameters in the Fontan group and confirmed that higher SPC flow/BSA was associated with higher BSA-adjusted ventricular EDV. Despite this association, higher SPC flow was not associated with other adverse laboratory or clinical markers, such as a lower ventricular ejection fraction, worse atrioventricular valve regurgitation, higher ventricular end-diastolic pressure, higher PA pressure, lower peak oxygen consumption, a higher rate of heart failure symptoms, and increased frequency of atrial or ventricular arrhythmias. It is likely that the progressive spontaneous decrease in SPC flow/BSA with increasing age may result in a minimal impact on long-term clinical status in most patients. These findings do not support the notion that SPC flow is an important contributor to long-term outcomes after Fontan surgery. However, we cannot exclude the possibility that SPC flow may have greater clinical impact in a small subset of patients with very large SPCs ${ }^{15}$ or in later adulthood.

In theory, SPC flow may be reduced by catheter-based occlusion, although the immediate and long-term efficacy of this procedure on quantitative SPC flow has not been studied. Our study was not designed to assess the efficacy of catheter-based occlusion of SPCs.

Nevertheless, in the Fontan group, despite a low rate of previous catheter-based occlusion of SPCs (13\%), SPC flow was relatively low and was not different in patients with or without histories of catheter-based occlusion of SPCs. In the pre-Fontan group, higher SPC flow was noted in patients who had undergone previous occlusion of SPCs, which possibly reflects persistently elevated residual flow despite SPC occlusion. It is possible that SPC flow in this group may have been even higher without prior catheter occlusion. Serial assessment of SPC flow before and after catheter occlusion and across palliative stages will be required to determine the effective- ness of catheter occlusion in mitigating SPC flow. 
No established reference method for quantifying SPC flow exists, and this has hindered investigation of the accuracy and validity of the newly described CMR techniques. Our finding that angiographic assessment of SPC flow grade correlated modestly with CMR measurements supports the validity of this new method for quantifying SPC flow. Although angiography does not permit the quantification of SPC flow, it is the technique used more frequently in the clinical setting to assess for the presence of SPCs. Challenges in angiographic assessment of SPC flow have been previously outlined, especially in patients with multiple small collateral vessels, ${ }^{1}$ and may be responsible for the significant overlap in SPC flow between angiographic SPC grades. In contrast, inaccuracies in estimation of SPC flow by CMR may exist due to technical factors and need to be explored, but such efforts will continue to be hindered by the lack of a reference technique. Further studies should be undertaken to determine if CMR estimation of SPC flow provides information incremental to conventional angiography and whether this additional information could be useful in refining the indications for catheter occlusion of SPCs and for monitoring the efficacy of this procedure.

Several limitations of this study are worth noting. The retrospective cross-sectional study design did not allow longitudinal assessment of the study cohort. The relatively modest sample size limited our ability to perform meaningful analyses of subgroups in the preFontan patients. The Fontan and pre-Fontan groups were somewhat dissimilar in terms of anatomic diagnoses, and this may have introduced bias into some of our analyses, although we were able to minimize bias by controlling for these differences in multivariate regression models. Because patients with large ferromagnetic artifacts due to stainless steel coils were excluded, there may have been a selection bias toward patients who did not undergo coil embolization of SPCs before 2002, when nonferromagnetic coils became standard practice at our institution. Furthermore, we only studied patients who were referred for CMR examinations, which may have introduced additional selection bias. It is important to recognize that the CMR technique used in this investigation cannot distinguish between SPC flow and flow through systemic-to-pulmonary venous collateral vessels. To minimize error related to this factor, we excluded patients with systemic-to-pulmonary venous collaterals visible on contrast magnetic resonance or $\mathrm{x}$-ray angiography

\section{References}

1. Triedman JK, Bridges ND, Mayer JE Jr, Lock JE. Prevalence and risk factors for aortopulmonary collateral vessels after Fontan and bidirectional Glenn procedures. J Am Coll Cardiol 1993;22:207215. [PubMed: 8509543]

2. Kanter KR, Vincent RN, Raviele AA. Importance of acquired system- ic-to-pulmonary collaterals in the Fontan operation. Ann Thorac Surg 1999;68:969-974. [PubMed: 10509993]

3. Bradley SM, McCall MM, Sistino JJ, Radtke WA. Aortopulmonary collateral flow in the Fontan patient: does it matter? Ann Thorac Surg 2001;72:408-415. [PubMed: 11515875]

4. Banka P, Sleeper LA, Atz AM, Cowley CG, Gallagher D, Gillespie MJ, Graham EM, Margossian R, McCrindle BW, Sang CJ, Williams IA, Newburger JW. Practice variability and outcomes of coil embolization of aortopulmonary collaterals before Fontan completion: a re- port from the Pediatric Heart Network Fontan Cross-Sectional Study. Am Heart J 2010;162:125-130.

5. Grosse-Wortmann L, Al-Otay A, Yoo S-J. Aortopulmonary collaterals after bidirectional cavopulmonary connection or fontan com- pletion: quantification with MRI. Circ Cardiovasc Imaging 2009; 2:219-225. [PubMed: 19808596] 
6. Whitehead KK, Gillespie MJ, Harris MA, Fogel MA, Rome JJ. Non- invasive quantification of systemic to pulmonary collateral flow: a major source of inefficiency in patients with superior cavopulmonary connections. Circ Cardiovasc Imaging 2009;2:405-411. [PubMed: 19808629]

7. Grosse-Wortmann L, Al-Otay A, Goo HW, Macgowan CK, Coles JG, Benson LN, Redington AN, Yoo SJ. Anatomical and functional evaluation of pulmonary veins in children by magnetic resonance imaging. J Am Coll Cardiol 2007;49:993-1002. [PubMed: 17336724]

8. Prakash A, Travison TG, Fogel MA, Hurwitz LM, Powell AJ, Printz BF, Puchalski MD, Shirali GS, Yoo SJ, Geva T. Relation of size of secondary ventricles to exercise performance in children after fontan operation. Am J Cardiol 2010;106:1652-1656. [PubMed: 21094369]

9. Alfakih K, Plein S, Thiele H, Jones T, Ridgway JP, Sivananthan MU. Normal human left and right ventricular dimensions for MRI as as- sessed by turbo gradient echo and steady-state free precession imaging sequences. J Magn Reson Imaging 2003;17:323-329. [PubMed: 12594722]

10. Rathod RH, Prakash A, Powell AJ, Geva T. Myocardial fibrosis identified by cardiac magnetic resonance late gadolinium enhancement is associated with adverse ventricular mechanics and ventricular tachy- cardia late after Fontan operation. J Am Coll Cardiol 2010;55:1721-1728. [PubMed: 20394877]

11. Paridon SM, Mitchell PD, Colan SD, Williams RV, Blaufox A, Li JS, Margossian R, Mital S, Russell J, Rhodes J. A cross-sectional study of exercise performance during the first 2 decades of life after the Fontan operation. J Am Coll Cardiol 2008;52:99-107 [PubMed: 18598887]

12. Bland JM, Altman DG. Statistical methods for assessing agreement be- tween two methods of clinical measurement. Lancet 1986;1:307-310. [PubMed: 2868172]

13. Ichikawa H, Yagihara T, Kishimoto H, Isobe F, Yamamoto F, Nishigaki K, Matsuki O, Fujita T. Extent of aortopulmonary collateral blood flow as a risk factor for Fontan operations. Ann Thorac Surg 1995;59: 433-437. [PubMed: 7847962]

14. McElhinney DB, Reddy VM, Tworetzky W, Petrossian E, Hanley FL, Moore P. Incidence and implications of systemic to pulmonary collaterals after bidirectional cavopulmonary anastomosis. Ann Thorac Surg 2000;69:1222-1228. [PubMed: 10800823]

15. Grosse-Wortmann L, Hamilton R, Yoo SJ. Massive systemic-to-pul-monary collateral arteries in the setting of a cavopulmonary shunt and pulmonary venous stenosis. Cardiol Young 2007;17:548-550 [PubMed: 17640400] 


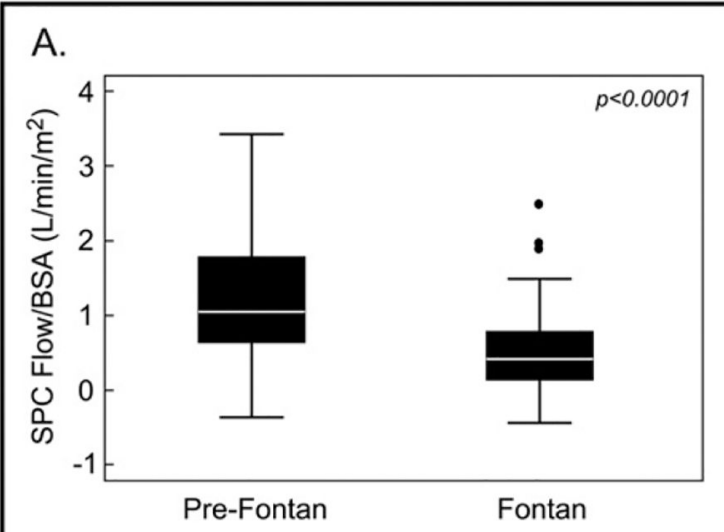

B.

Figure 1.

SPC flow in Fontan and pre-Fontan patients. SPC flow/BSA ( $A$ ) and SPC flow/ascending aortic flow $(B)$ were higher in pre-Fontan patients. 
A.

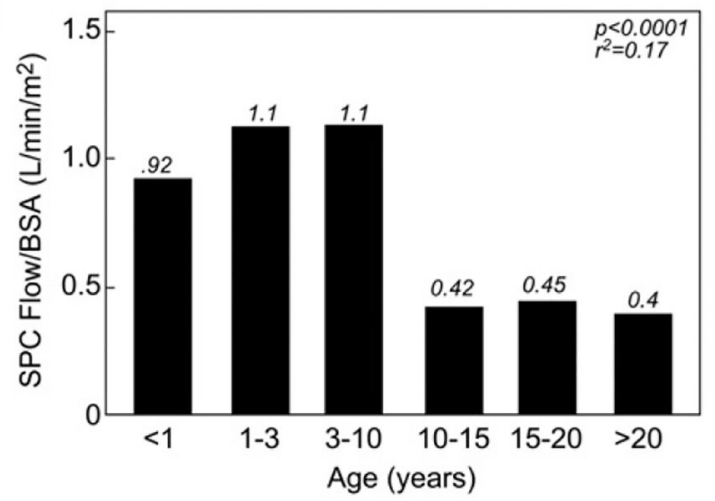

B.

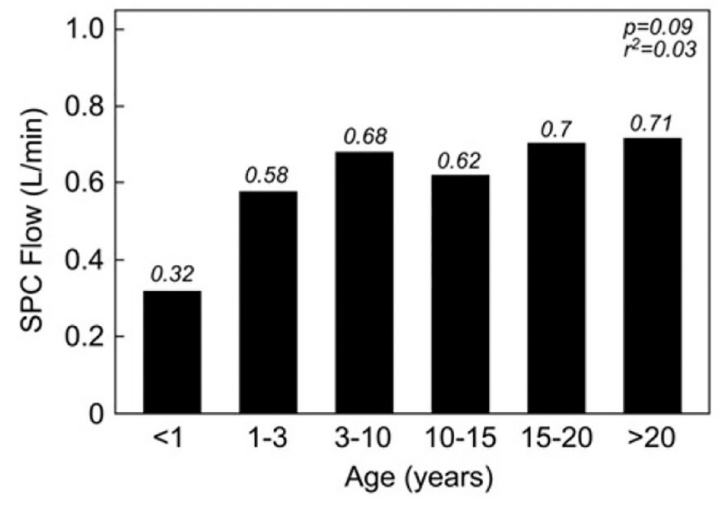

Figure 2.

Median SPC flow by age in all patients. (A) SPC flow/BSA decreased nonlinearly with increasing age. (B) In contrast, absolute SPC flow does not vary significantly with increasing age 


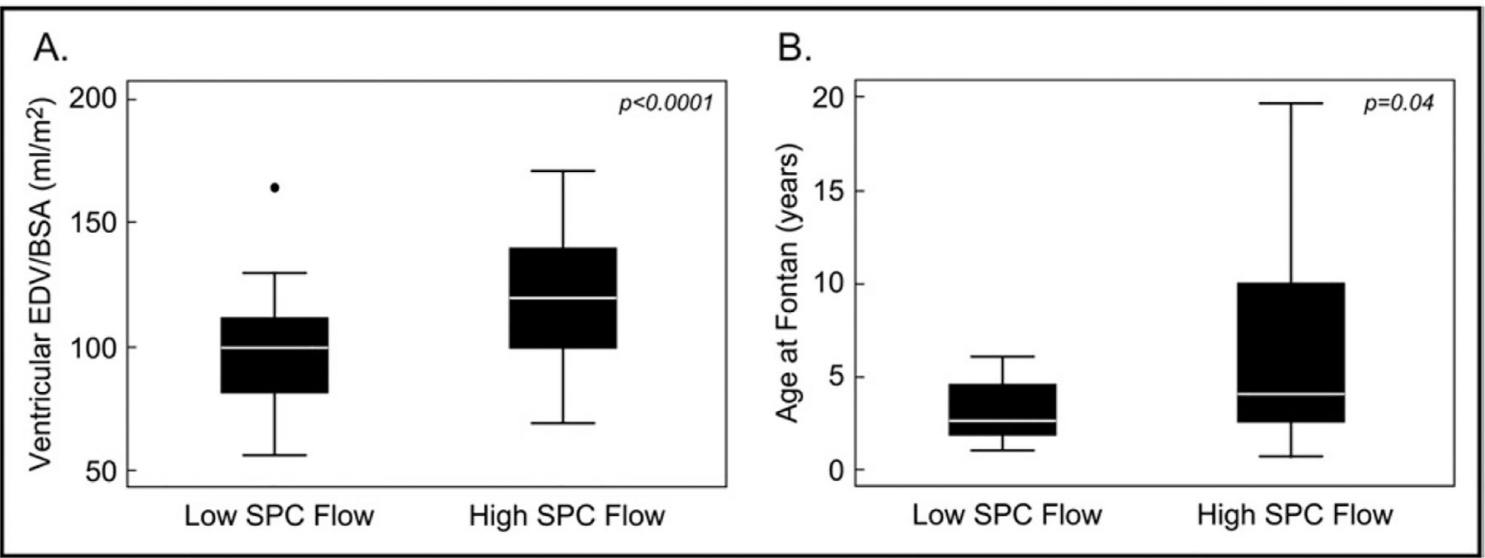

Figure 3.

Comparison between patients in the highest and lowest quartiles of SPC flow in Fontan group. Compared to patients in the lowest quartile of SPC flow (low SPC flow), those in the higher quartile (high SPC flow) had larger ventricular EDVs $(A)$ and had undergone the Fontan operation at an older age $(B)$. Because values for age at Fontan surgery were skewed, logarithmic transformation was applied before comparison between groups 


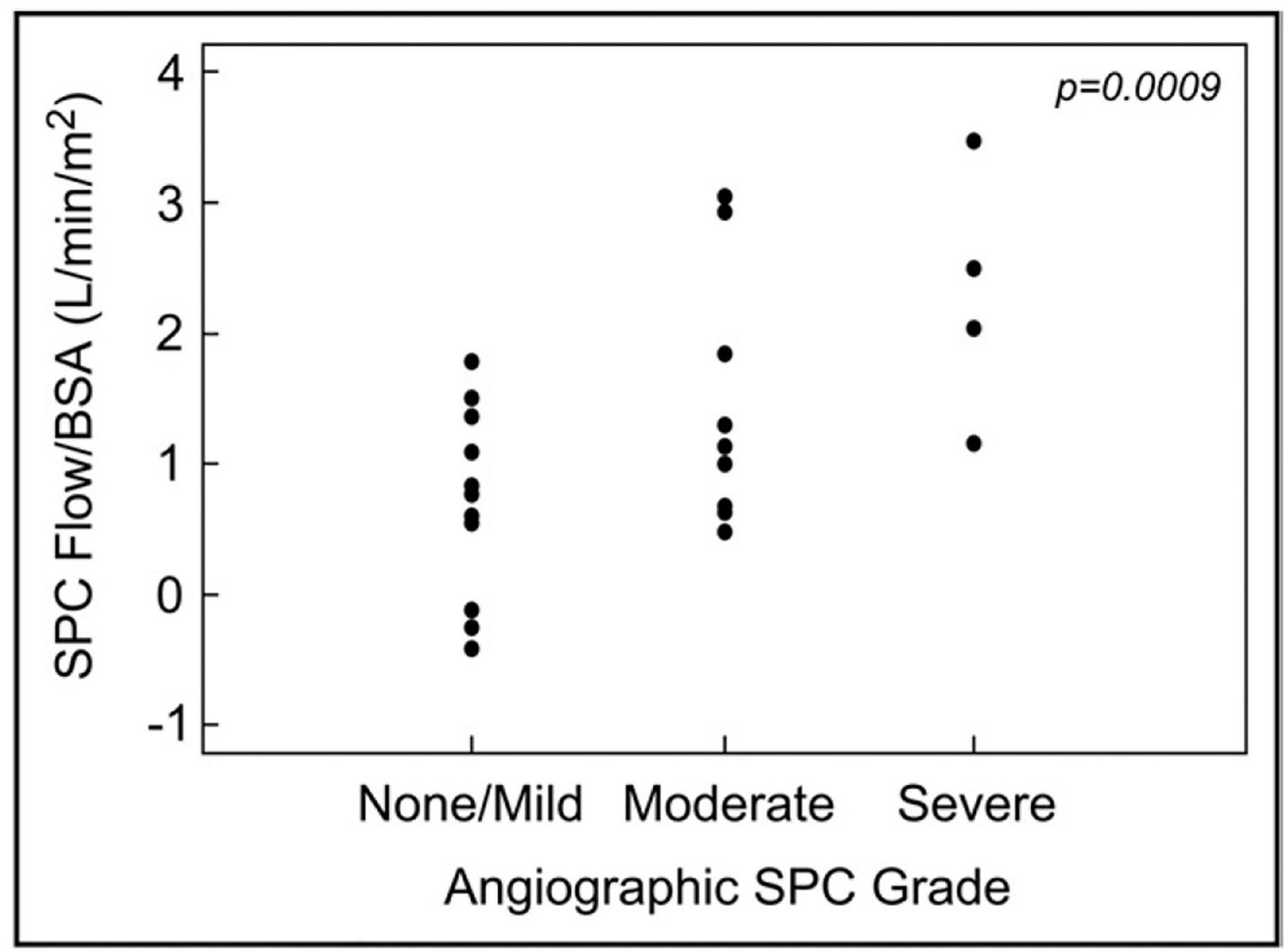

Figure 4.

CMR SPC flow/BSA and angiographic SPC grade in the pre- Fontan group $(n=26)$. SPC flow was higher in patients with higher angiographic SPC grade but with significant overlap between grades. 


\section{Table 1}

Angiographic systemic-to-pulmonary collateral artery grading

\begin{tabular}{ll}
\hline Grade & \multicolumn{1}{c}{ Description } \\
\hline None/mild & None or faint opacification of PAs and/or pulmonary veins on aortography or subclavian artery angiography; <1 lobe in each lung \\
Moderate & $\begin{array}{l}\text { Moderate opacification of PAs and/or pulmonary veins in both lungs on aortography or subclavian artery angiography; }>1 \text { lobe; } \\
\text { vessels opacified after }>2 \text { cardiac cycles; extensive filling of PAs or pulmonary veins in a single lobe or lung; pulmonary vessels } \\
\text { opacified within } 1 \text { to } 2 \text { cardiac cycles }\end{array}$ \\
& $\begin{array}{l}\text { Extensive filling of PAs and/or pulmonary veins on aortography in multiple lobes of both lungs; pulmonary vessels opacified } \\
\text { within } 1 \text { to } 2 \text { cardiac cycles, usually from multiple systemic arterial sources }\end{array}$
\end{tabular}
within 1 to 2 cardiac cycles, usually from multiple systemic arterial sources 
Table 2

Characteristics of the Fontan group

\begin{tabular}{|c|c|c|c|c|}
\hline \multirow[t]{2}{*}{ Variable } & \multirow[t]{2}{*}{ All Patients $(n=78)$} & \multicolumn{2}{|c|}{ SPC Flow $\left(\mathrm{L} / \mathbf{m i n} / \mathbf{m}^{2}\right)$} & \multirow[t]{2}{*}{ p Value } \\
\hline & & Low $(<0.43)(n=39)$ & High $(>0.43)(n=39)$ & \\
\hline Age at enrollment (years) & $18.6(5-53)$ & $21.1(5-53)$ & $17.6(5-37)$ & 0.09 \\
\hline Female (\%) & $28(36 \%)$ & $15(38 \%)$ & $13(33 \%)$ & 0.63 \\
\hline Time after Fontan surgery (years) & $15.9 \pm 7$ & $16.7 \pm 6$ & $14.1 \pm 6$ & 0.08 \\
\hline Previous systemic-to-PA shunt & $61(78 \%)$ & $32(82 \%)$ & $29(74 \%)$ & 0.4 \\
\hline Previous Glenn shunt & $35(45 \%)$ & $14(36 \%)$ & $21(54 \%)$ & 0.11 \\
\hline Age at volume unloading surgery (years) & $0.8(0.3-21)$ & $0.84(0.5-20.6)$ & $0.75(0.3-13.2)$ & 0.38 \\
\hline Age at Fontan surgery (years) & $3.6(1-40)$ & $3.9(1-40)$ & $3.2(1-20)$ & 0.36 \\
\hline Previous catheter occlusion of SPCs & $10(13 \%)$ & $3(8 \%)$ & $7(18 \%)$ & 0.18 \\
\hline Anatomic diagnosis & & & & 0.36 \\
\hline Tricuspid atresia & $26(33 \%)$ & $9(23 \%)$ & $17(44 \%)$ & \\
\hline Double-outlet right ventricle & $18(23 \%)$ & $9(23 \%)$ & $9(23 \%)$ & \\
\hline HLHS & $12(15 \%)$ & $7(18 \%)$ & $5(13 \%)$ & \\
\hline $\begin{array}{l}\text { L-loop transposition of the great arteries or double- } \\
\text { outlet right ventricle with pulmonary stenosis/atresia }\end{array}$ & $7(9 \%)$ & $4(10 \%)$ & $3(8 \%)$ & \\
\hline Double-inlet left ventricle & $6(8 \%)$ & $5(13 \%)$ & $1(3 \%)$ & \\
\hline Hypoplastic tricuspid valve/right ventricle & $4(5 \%)$ & $2(5 \%)$ & $2(5 \%)$ & \\
\hline Pulmonary atresia, intact ventricular septum & $3(4 \%)$ & $1(3 \%)$ & $2(5 \%)$ & \\
\hline Unbalanced atrioventricular canal & $2(3 \%)$ & $2(3 \%)$ & - & \\
\hline Type of Fontan operation & & & & 0.33 \\
\hline Lateral tunnel & $50(64 \%)$ & $25(64 \%)$ & $25(64 \%)$ & \\
\hline Right atrium to PA & $22(28 \%)$ & $13(33 \%)$ & $9(23 \%)$ & \\
\hline Right atrium-to-right ventricle conduit & $4(5 \%)$ & $2(5 \%)$ & $2(5 \%)$ & \\
\hline Extracardiac conduit & $2(3 \%)$ & $1(3 \%)$ & $1(3 \%)$ & \\
\hline Systemic oxygen saturation at rest $(\%)$ & $93.7 \pm 3$ & $94.1 \pm 3$ & $93.3 \pm 3$ & 0.24 \\
\hline Heart failure symptoms & $21(27 \%)$ & $10(26 \%)$ & $11(28 \%)$ & 0.80 \\
\hline$\%$ predicted peak oxygen consumption $(n=43)$ & $59.2 \pm 14.4$ & $56.7 \pm 14$ & $61.3 \pm 15$ & 0.30 \\
\hline Ventricular EDV/BSA $\left(\mathrm{ml} / \mathrm{m}^{2}\right)^{*}$ & $104.7 \pm 35$ & $94.5 \pm 27$ & $115.2 \pm 40$ & 0.015 \\
\hline Ventricular ejection fraction (\%) & $53.9 \pm 16$ & $55.4 \pm 9$ & $52.3 \pm 11$ & 0.19 \\
\hline $\begin{array}{l}\text { More than mild atrioventricular valve regurgitation by } \\
\text { echocardiography }\end{array}$ & $22(18 \%)$ & $6(15 \%)$ & $7(16 \%)$ & 0.19 \\
\hline History of any arrhythmia & $54(69 \%)$ & $25(64 \%)$ & $29(74 \%)$ & 0.33 \\
\hline Atrial arrhythmia & $44(56 \%)$ & $21(54 \%)$ & $23(59 \%)$ & 0.65 \\
\hline Ventricular arrhythmia & $22(28 \%)$ & $10(26 \%)$ & $12(31 \%)$ & 0.62 \\
\hline Ventricular end-diastolic pressure $(\mathrm{mm} \mathrm{Hg})(\mathrm{n}=14)$ & $9.2 \pm 4$ & $9.9 \pm 5$ & $8.3 \pm 3$ & 0.50 \\
\hline Mean PA pressure $(\mathrm{mm} \mathrm{Hg})(\mathrm{n}=14)$ & $13.6 \pm 4$ & $14 \pm 3$ & $13.2 \pm 3$ & 0.60 \\
\hline Unilateral branch PA stenosis on CMR imaging ${ }^{\dagger}$ & $4(5 \%)$ & $1(3 \%)$ & $3(8 \%)$ & 0.60 \\
\hline
\end{tabular}


Greater than 50\% narrowing and $<30 \%$ differential lung flow 


\section{Table 3}

Characteristics of the pre-Fontan group

\begin{tabular}{|c|c|c|c|c|}
\hline \multirow[t]{2}{*}{ Variable } & \multirow[t]{2}{*}{ All Patients $(\mathrm{n}=\mathbf{3 8})$} & \multicolumn{2}{|c|}{ SPC Flow $\left(L / \mathbf{m i n} / \mathbf{m}^{2}\right)$} & \multirow[t]{2}{*}{ p Value } \\
\hline & & Low $(<1.06)(n=19)$ & High $(>1.06)(n=19)$ & \\
\hline Age at enrollment (years) & $2.01(0.2-15)$ & $1.19(0.2-7)$ & $2.32(0.3-15)$ & 0.10 \\
\hline Female $(\%)$ & $14(37 \%)$ & $7(37 \%)$ & $7(37 \%)$ & 1 \\
\hline Previous systemic-to-PA shunt & $21(55 \%)$ & $6(40 \%)$ & $15(79 \%)$ & 0.008 \\
\hline Previous bidirectional Glenn shunt & $28(74 \%)$ & $11(58 \%)$ & $17(90 \%)$ & 0.06 \\
\hline Age at volume unloading surgery (years) & $0.5(0.2-8)$ & $0.55(0.3-5)$ & $0.5(0.2-8)$ & 0.60 \\
\hline Previous catheter occlusion of SPCs & $15(39 \%)$ & $2(11 \%)$ & $13(68 \%)$ & 0.001 \\
\hline Anatomic diagnosis & & & & 0.90 \\
\hline HLHS & $20(53 \%)$ & $9(47 \%)$ & $11(58 \%)$ & \\
\hline Unbalanced atrioventricular canal & $7(18 \%)$ & $4(21 \%)$ & $3(16 \%)$ & \\
\hline Tricuspid atresia & $6(16 \%)$ & $3(16 \%)$ & $3(16 \%)$ & \\
\hline Double-outlet right ventricle & $3(8 \%)$ & $2(11 \%)$ & $1(5 \%)$ & \\
\hline Hypoplastic tricuspid valve/right ventricle & $2(5 \%)$ & $1(5 \%)$ & $1(5 \%)$ & \\
\hline Mean systemic oxygen saturation (\%) & $80.5 \pm 5$ & $79.5 \pm 5$ & $81.4 \pm 6$ & 0.80 \\
\hline Ventricular EDV/BSA $\left(\mathrm{ml} / \mathrm{m}^{2}\right)(\mathrm{n}=29)^{*}$ & $121.5 \pm 34$ & $111.3 \pm 25$ & $132.4 \pm 39$ & 0.09 \\
\hline Ventricular ejection fraction $(\%)$ & $54.6 \pm 10$ & $54.0 \pm 4$ & $55.1 \pm 4$ & 0.9 \\
\hline Unilateral branch PA stenosis on $\mathrm{CMR}^{\dagger}$ & $5(13 \%)$ & $1(5 \%)$ & $4(21 \%)$ & 0.3 \\
\hline $\begin{array}{l}\text { More than mild atrioventricular valve regurgitation by } \\
\text { echocardiography }\end{array}$ & $7(18 \%)$ & $4(21 \%)$ & $3(16 \%)$ & 0.99 \\
\hline Restrictive atrial septal defect ${ }^{\frac{t^{t}}{4}}$ & $6(16 \%)$ & $1(5 \%)$ & $5(26 \%)$ & 0.18 \\
\hline $\begin{array}{l}\text { Ventricular end-diastolic or pulmonary capillary wedge } \\
\text { pressure }(\mathrm{mm} \mathrm{Hg})(\mathrm{n}=27)^{\mathcal{S}}\end{array}$ & $8.9 \pm 2.0$ & $8.3 \pm 2$ & $9.5 \pm 2.0$ & 0.18 \\
\hline Mean PA pressure $(\mathrm{mm} \mathrm{Hg})(\mathrm{n}=14)$ & $13.8 \pm 3$ & $13 \pm 3$ & $14.5 \pm 3$ & 0.15 \\
\hline \multicolumn{5}{|c|}{ Data are expressed as median (range), number (percentage), or mean $\pm \mathrm{SD}$. } \\
\hline \multicolumn{5}{|c|}{ Excluding patients with more than mild valve regurgitation. } \\
\hline \multicolumn{5}{|c|}{ Greater than 50\% narrowing and <30\% differential lung flow. } \\
\hline Mean gradient $>3 \mathrm{~mm} \mathrm{Hg}$ by catheterization. & & & & \\
\hline xcluding patients with restrictive atrial septal defects. & & & & \\
\hline
\end{tabular}




\section{Table 4}

Cardiac magnetic resonance imaging flow data

\begin{tabular}{lccc}
\hline & Fontan & Pre-Fontan & p Value \\
\hline Superior vena cava flow $\left(\mathrm{L} / \mathrm{min} / \mathrm{m}^{2}\right)$ & $0.9 \pm 0.3(\mathrm{n}=61)$ & $1.94 \pm 0.85(\mathrm{n}=38)$ & $<0.0001$ \\
Inferior vena cava flow $\left(\mathrm{L} / \mathrm{min} / \mathrm{m}^{2}\right)$ & $1.55 \pm 0.5(\mathrm{n}=58)$ & $1.41 \pm 0.57(\mathrm{n}=36)$ & 0.20 \\
Systemic blood flow (total caval flow) & $2.4 \pm 0.63(\mathrm{n}=78)$ & $3.5 \pm 1.01(\mathrm{n}=36)$ & $<0.0001$ \\
Inferior vena cava flow/total caval flow & $0.63 \pm 0.11$ & $0.42 \pm 0.14$ & $<0.0001$ \\
Descending aorta flow $\left(\mathrm{L} / \mathrm{min} / \mathrm{m}^{2}\right)$ & $1.64 \pm 0.49(\mathrm{n}=45)$ & $1.24 \pm 0.4(\mathrm{n}=29)$ & 0.001 \\
Ascending aorta flow $\left(\mathrm{L} / \mathrm{min} / \mathrm{m}^{2}\right)$ & $2.92 \pm 0.7(\mathrm{n}=78)$ & $4.67 \pm 1.32(\mathrm{n}=38)$ & $<0.0001$ \\
Branch PA flow & & & \\
Right $\left(\mathrm{L} / \mathrm{min} / \mathrm{m}^{2}\right)$ & $1.44 \pm 0.5(\mathrm{n}=55)$ & $1.33 \pm 0.51(\mathrm{n}=32)$ & 0.34 \\
Left $\left(\mathrm{L} / \mathrm{min} / \mathrm{m}^{2}\right)$ & $0.97 \pm 0.4(\mathrm{n}=62)$ & $0.73 \pm 0.35(\mathrm{n}=32)$ & 0.009 \\
Right $/(\mathrm{right}+$ left $)$ & $0.6 \pm 0.1$ & $0.64 \pm 0.14$ & 0.14 \\
\hline
\end{tabular}

